\title{
Interception tissulaire au moyen de volets et greffes osseuses dans le traitement chirurgical des volumineux kystes maxillaires : étude clinique sur 10 patients
}

\author{
Baumann B, Ridoux C, Saez P. \\ (Centre Hospitalier de Mulhouse)
}

But de la présentation

Les approches chirurgicales permettant l'énucléation des volumineux kystes des maxillaires $(\leq 3 \mathrm{~cm})$ ont été largement décrites (Tarragano et coll., 2010). Avec les progrès de l'imagerie, les techniques et les voies d'abords ont su évoluer. Les reconstructions tri dimensionnelles issues des acquisitions scanners ont permis de connaître les rapports anatomiques exacts de ces processus tumoraux avec les structures avoisinantes et ainsi de choisir la technique opératoire la mieux adaptée et la moins invasive.

L'objectif de la présentation sera d'illustrer au moyen de cas cliniques une nouvelle technique chirurgicale mise en œuvre : I'utilisation de volets et greffes osseuses.

\section{Matériel et Méthodes}

La chirurgie des bases osseuses maxillaires ne doit pas hypothéquer une possibilité de chirurgie reconstructrice ou une réhabilitation future de la cavité buccale. En chirurgie orale, la préservation des volumes osseux est capitale (Seban et coll., 2008).

A partir de 10 cas cliniques nous avons comparé nos résultats à ceux de la littérature. Deux difficultés récurrentes dans le traitement chirurgical des volumineux processus kystiques des maxillaires apparaissent : la perte significative de volume osseux et l'invagination des tissus mous.

Ce dernier paramètre semble le plus difficile à gérer ; l'invagination muqueuse au cours de la cicatrisation peut alors compromettre la qualité de vie de nos patients (Atwood ; 1963.).

\section{Résultats}

Une nouvelle approche chirurgicale consiste en l'utilisation de volets osseux pour mettre à jour le site opératoire et réaliser l'énucléation kystique. Le repositionnement in fine du volet ou le recours à une greffe osseuse pendant le même temps opératoire permet ensuite de guider la cicatrisation, voire d'augmenter les volumes osseux. A partir d'une étude de 10 patients et à partir de la littérature nous tenterons de proposer un arbre décisionnel pour guider le choix opératoire.

\section{Conclusion}

La présentation aura pour objectif de décrire une technique chirurgicale permettant l'interception tissulaire pour le repositionnement des tissus mous lors de l'énucléation de volumineux processus kystiques. Le recours aux volets ou aux greffes osseuses permet désormais de reconstruire les volumes osseux perdus et guide la cicatrisation des tissus de recouvrement. 